\title{
Bifunctional apoptosis inhibitor (BAR) protects neurons from diverse cell death pathways
}

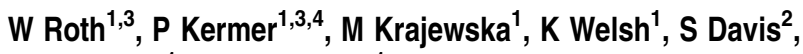 \\ S Krajewski ${ }^{1}$ and JC Reed*,1 \\ 1 The Burnham Institute, 10901 N. Torrey Pines Road, La Jolla, CA 92037, USA \\ ${ }^{2}$ Isis Pharmaceuticals, Inc., Carlsbad, CA, USA \\ ${ }^{3}$ WR and PK contributed equally to this work. \\ ${ }^{4}$ Current address: Department of Neurology, University of Goettingen, \\ Germany. \\ * Corresponding author: JC Reed, Tel: 858-646-3140; Fax: 858-646-3194; \\ E-mails: jreed@burnham.org, reedoffice@burnham.org
}

Received 24.2.03; revised 10.4.03; accepted 07.5.03

\begin{abstract}
The bifunctional apoptosis regulator (BAR) is a multidomain protein that was originally identified as an inhibitor of Baxinduced apoptosis. Immunoblot analysis of normal human tissues demonstrated high BAR expression in the brain, compared to low or absent expression in other organs. Immunohistochemical staining of human adult tissues revealed that the BAR protein is predominantly expressed by neurons in the central nervous system. Immunofluorescence microscopy indicated that BAR localizes mainly to the endoplasmic reticulum (ER) of cells. Overexpression of BAR in CSM 14.1 neuronal cells resulted in significant protection from a broad range of cell death stimuli, including agents that activate apoptotic pathways involving mitochondria, TNFfamily death receptors, and ER stress. Downregulation of BAR by antisense oligonucleotides sensitized neuronal cells to induction of apoptosis. Moreover, the search for novel interaction partners of BAR identified several candidate proteins that might contribute to the regulation of neuronal apoptosis (HIP1, Hippi, and Bap31). Taken together, the expression pattern and functional data suggest that the BAR protein is involved in the regulation of neuronal survival. Cell Death and Differentiation (2003) 10, 1178-1187. doi:10.1038/ sj.cdd. 4401287
\end{abstract}

Keywords: apoptosis; BAR; neurodegeneration; neurons; astrocytoma

Abbreviations: Bap31, B-lymphocyte receptor-associated protein 31; BAR, bifunctional apoptosis regulator; BID, BH3 interacting domain death agonist; CNS, central nervous system; DED, death effector domain; ER, endoplasmic reticulum; FADD, Fasassociated death domain; FITC, fluorescein isothiocyanate; GFP, green fluorescent protein; HIP1, Huntingtin-interacting protein; Hippi, HIP1 protein interactor; kDa, kilo Dalton; MPTP, 1-methyl4-phenyl-1,2,3,6-tetrahydropyridine; PBS, phosphate-buffered saline; kb, kilobase pairs; PDI, protein disulfide isomerase;
PEA15, phosphoprotein enriched in astrocytes 15; STS, staurosporine; XIAP, X-linked inhibitor of apoptosis

\section{Introduction}

Tight regulation of signalling pathways controlling neuronal death and survival is crucial for the normal development and function of the nervous system. In contrast to most cell types, neurons survive for the entire lifetime of the organism and therefore need to possess powerful intracellular mechanisms to antagonize cell death stimuli. Dysregulation of apoptotic cell death pathways can lead to several neurological conditions. Neurodegenerative diseases such as Parkinson's or Huntington's disease are characterized by a relative preponderance of proapoptotic intracellular signalling events in certain populations of neurons. ${ }^{1,2}$ Similarly, delayed neuronal death in the ischemic penumbra after occurrence of a stroke may be mediated by apoptosis. ${ }^{3}$

Several antiapoptotic proteins are involved in the protection of neurons from damaging influences such as oxidative stress, lack of neurotrophic factors, or toxins. Antiapoptotic members of the $\mathrm{Bcl}-2$ family have been shown to protect neurons from metabolic and oxidative insults by stabilizing mitochondrial functions, thereby inhibiting cytochrome $c$ release, Apaf-1 oligomerization, and activation of Caspase 9. ${ }^{4}$ Inhibitor of apoptosis proteins (IAPs) may play an important role in neuronal cell survival mechanisms by directly binding to and inhibiting Caspases 3, 7, or 9. ${ }^{5,6}$ Compared to the regulation of mitochondrial apoptosis, the relevance of death receptor- and Caspase-8-mediated apoptosis in neurons is far less well documented. However, TNF $\alpha$ may induce neuronal death after nerve growth factor withdrawal ${ }^{7}$ and HIV1 infection. ${ }^{8,9}$ Further, neuronal death in Huntington's disease may involve Caspase-8 activation dependent on a protein complex comprising HIP1, Hippi, and Caspase-8. ${ }^{10}$

The bifunctional apoptosis inhibitor (BAR) is a multidomain protein that was first discovered as an inhibitor of Bax-induced cell death. ${ }^{11}$ BAR is capable of inhibiting apoptosis induced by TNF-family death receptors ('extrinsic pathway') as well as mitochondria-dependent apoptosis ('intrinsic pathway'). Interaction of BAR with $\mathrm{Bcl}-2$ or $\mathrm{Bcl}-\mathrm{X}_{\mathrm{L}}$ via a SAM domain may contribute to the antiapoptotic properties of BAR. Moreover, the BAR protein contains a domain that shares similarity with classical death effector domains (termed 'pseudo DEDs'), which mediates Caspase- 8 binding. Therefore, BAR was suggested to act as a scaffold protein that can bridge components of extrinsic and intrinsic apoptosis pathways. This theory was supported by the finding that active Caspase8 subunits are sequestered to $\mathrm{Bcl}-\mathrm{X}_{\mathrm{L}} / \mathrm{BAR}$ complexes, thereby preventing further cleavage of Caspase- 8 substrates and subsequent cell death in the context of Fas-induced apoptosis. $^{12}$

In this report, we provide evidence that BAR is mainly expressed in vivo by neurons in the central and peripheral 
nervous systems. Overexpressed BAR protects neuronal cell lines from a broad range of cell death stimuli, while downregulation of endogenous BAR leads to sensitization to apoptosis. Our findings suggest that the biological functions of the BAR protein in vivo are likely to be relevant to suppression of cell death and maintenance of survival of neurons.

\section{Results}

\section{BAR is predominantly expressed in neurons}

The physiological functions of the BAR protein are largely unknown. To investigate the possible roles of BAR in vivo, we studied the expression pattern of this protein in human organs. To this end, we performed an immunoblot analysis of human normal tissue samples using a BAR-specific rabbit antiserum, previously described. ${ }^{11}$ BAR expression was highest by far in brain, with moderate expression in small intestine, weak expression in testes, and only faint expression in liver and skeletal muscle (Figure 1a). No signal was detected in heart, kidney, lung, and spleen.

To demonstrate antibody specificity, we analyzed tissue lysates of brain, heart, and small intestine side by side with lysates of SK-N-BE(2) neuroblastoma cells that had been transfected with BAR-specific antisense oligonucleotides (Figure 1b). Treatment of SK-N-BE(2) cells with BAR-specific antisense oligonucleotides resulted in a significant decrease in signal intensity of an $\sim 50 \mathrm{kDa}$ protein band that comigrates in gels with the same immunoreactive band as present endogenously in tissue lysates, confirming that this band is BAR.

Owing to the distinct expression pattern in various organs, we studied the anatomic distribution of BAR in greater detail by performing immunohistochemical staining of paraffinembedded, human tissue specimens. The staining pattern

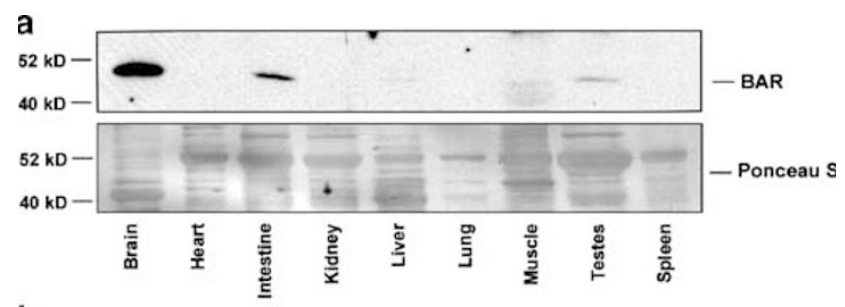

b

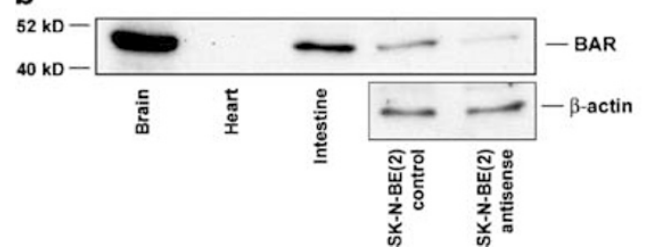

Figure 1 Expression of BAR in human adult tissues. (a) Immunoblot analysis of BAR in human tissues. Human tissue lysates $(25 \mu \mathrm{g})$ were analyzed by SDSPAGE/immunoblot using anti-BAR antiserum $(1: 3000)$ and HRP-coupled secondary antibody (top panel). As a protein loading control, the membrane was stained with Ponceau $S$ solution (bottom panel). (b) Specificity of the polyclonal anti-BAR antibody was demonstrated by including cell lysates from anti-BAR antisense oligonucleotide-treated SK-N-BE(2) cells during immunoblot analysis of human tissue samples. The BAR protein migrates at a molecular weight of $\sim 50 \mathrm{kDa}$ was generally organellar or diffuse cytosolic. Staining for BAR was present in keratinizing epithelium in the upper layers of the epidermis (Figure 2a), but not in nonkeratinizing squamous epithelium of the esophagus (Figure 2b), cervix, stomach, intestine, and lung (not shown). Moreover, BAR expression was found in seminiferous tubules (secondary spermatocytes and early spermatids; Figure 2c), in endometrial glands, the fallopian tubes, and in plasma cells in the colonic lamina propria (not shown). Multiple tissues were entirely negative for BAR staining including prostate (Figure 2d), spleen, bone marrow, smooth and skeletal muscle, hypophysis, thyroid, liver, and pancreas.

Overall, BAR staining was most prominent in the nervous system. Highest signal intensities were observed in neurons in the central and peripheral nervous systems. Both the cell bodies and axons of neurons were stained with anti-BAR antibody. Among neuronal types, the strongest BAR immunostaining was found in pyramidal neurons located in cortical layers III-V (Figure $2 \mathrm{e}-\mathrm{h}$ ) and in the neurons of the nuclei of pontomedullary reticular formation, such as the principal olivary nucleus (Figure 2i,j) and the facial (Figure 2k) and hypoglossal nuclei, and in the red nucleus in the mesencephalon. Purkinje cells, granular neurons, and the synapses of the mossy fibers on granule cells in the cerebellum were strongly positive (Figure 2l).

Within the peripheral nervous system, neurons in the sympathetic ganglia and dorsal root ganglia (Figure $2 \mathrm{~m}-\mathrm{o}$ ) exhibited strong staining. Moreover, peripheral neurons showed intense staining in ganglion cells of Meissner's plexus, interspersed among negative smooth muscle adjoining unstained gastrointestinal epithelium (not shown). In contrast to the adrenal cortex, where no staining was observed, neuroendocrine chromaffin cells in the adrenal medulla were positive for BAR staining (Figure $2 p-r$ ). Strong BAR signal was also observed in pinealocytes, but not in the glial compartment (Figure 2s,t).

\section{BAR localizes to the endoplasmic reticulum (ER)}

Since immunohistochemistry showed an organellar-like staining pattern for BAR in neuronal cells, we examined the subcellular localization of the BAR protein in cells of neural origin. First, we sought to confirm the staining pattern observed in immunohistochemistry by performing immunofluorescence staining of SK-N-BE(2) neuroblastoma cells that had been shown to express endogenous BAR (Figure $1 \mathrm{~b}$ ). In accordance with the immunohistochemical findings, staining of these cells with anti-BAR antiserum produced a perinuclear and organellar-like fluorescence signal, whereas staining with preimmune serum produced no signal (Figure $3 a, b)$. To examine BAR localization in more detail, we stably transfected the rat nigrostriatal cell line CSM 14.1 and the human astrocytoma cell line LN18 with a truncated version of BAR lacking its N-terminal RING domain, $\operatorname{BAR}(\Delta R)$. Removal of the RING domain of BAR has been shown to prevent proteasome-dependent degradation of the protein, ${ }^{11}$ allowing greater protein accumulation. The generation of these cell lines served as a useful model for expression and functional studies since: (i) BAR-transfected CSM cells allow the study of BAR localization and function in cells of neuronal origin, while 

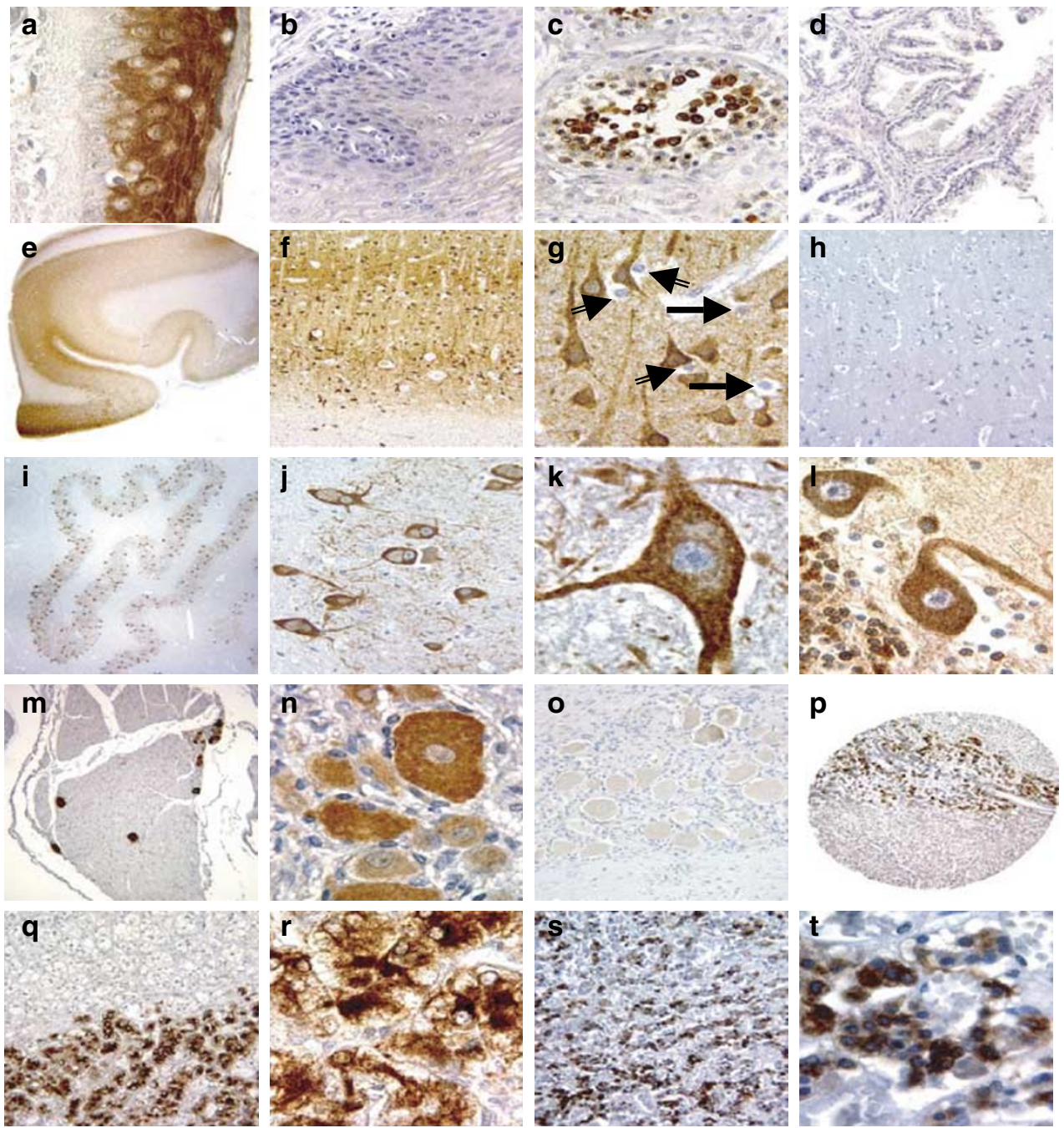

Figure 2 Immunohistochemical analysis of BAR expression. Expression of the BAR protein in normal human tissues is demonstrated in epidermis $((\mathbf{a}) \times 400)$, esophageal squamous, nonkeratinizing epithelium $((\mathbf{b}) \times 200)$, testis $((\mathbf{c}) \times 400)$, and prostate $((\mathbf{d}) \times 200)$. In the central nervous system, BAR expression was exclusively neuronal as shown in the examples from the occipital cortex $(\mathbf{e}) \times 40 ;(\mathbf{f}) \times 100 ;(\mathbf{g}) \times 400)$. The subcellular pattern in neurons is organellar and fine granular cytoplasmic in perikaryon and dendrites. The negative astroglia (long arrows) and satellite oligodendroglia (short arrows) are clearly visible in panel (g). The specificity control of the antibody preabsorbed with BAR recombinant protein is demonstrated in $(h)(\times 100)$ and $(\mathbf{o})(\times 400)$. BAR immunoreactivity in other parts of the CNS is shown in examples of olivary nucleus $((\mathbf{i}) \times 100$ and $(\mathbf{j}) \times 400)$, motoneurons in the facial nucleus of the brain stem $((\mathbf{k}) \times 1000)$, and Purkinje cells in the cerebellum $((\mathbf{I}) \times 1000)$. In the peripheral nervous system, ganglion cells in dorsal root ganglia were strongly positive for BAR staining $((\mathbf{m}) \times 100$ and $(\mathbf{n}) \times 1000)$, whereas nerve fibers and myelin were negative. Moreover, prominent BAR immunoreactivity was detected in adrenal medulla $((\mathbf{p}) \times 80 ;(\mathbf{q}) \times 200 ;(\mathbf{r}) \times 1000)$ and in pinealocytes $((\mathbf{s}) \times 200 ;(\mathbf{t}) \times 1000)$

BAR-transfected astrocytic LN18 cells serve as a control cell line for possible neuron-specific effects; and (ii) the epitopetagged, ectopically expressed BAR protein allows detection by two independent antibodies, anti-BAR and anti-myc. For each cell line, several subclones of BAR transfectants were generated (Figure 3c). Cells stably transfected with empty expression vectors served as controls. The subcellular localization of the epitope-tagged BAR in these transfected cells was then accomplished by immunofluorescence and organelles were simultaneously colocalized using a mitochondrial marker (Mitotracker) or an ER marker (anti-protein disulfide isomerase, PDI). As shown in Figure 3, BAR exhibited clear colocalization with PDI (Figure $3 d-f$ ), whereas only partial overlap was detected with the mitochondrial marker (Figure 3g-i). Similar findings were obtained in LN18 cells, as well as in transiently transfected COS-7 cells (data not shown), suggesting that BAR resides mainly in association with the ER, independent of cell type.

\section{BAR protects neuronal cells from a broad range of cell death stimuli}

The prominent expression of BAR in neurons together with its distinct subcellular localization suggests that BAR might be relevant for survival pathways, particularly in CNS cells. Therefore, we sought to characterize BAR functions in CSM neuronal cells and to compare these results with BAR-induced effects in other, non-neuronal CNS cells. Since BAR was initially identified as an apoptosis regulator that inhibits intrinsic as well as extrinsic apoptotic signalling, we first 

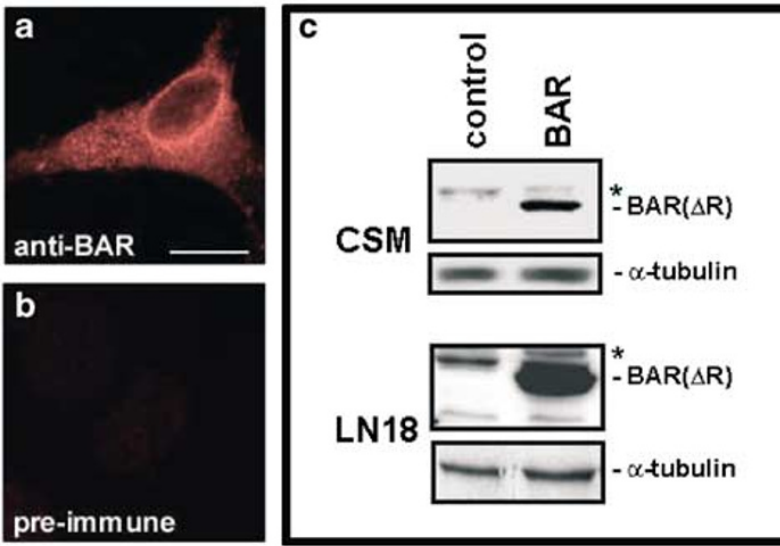

c
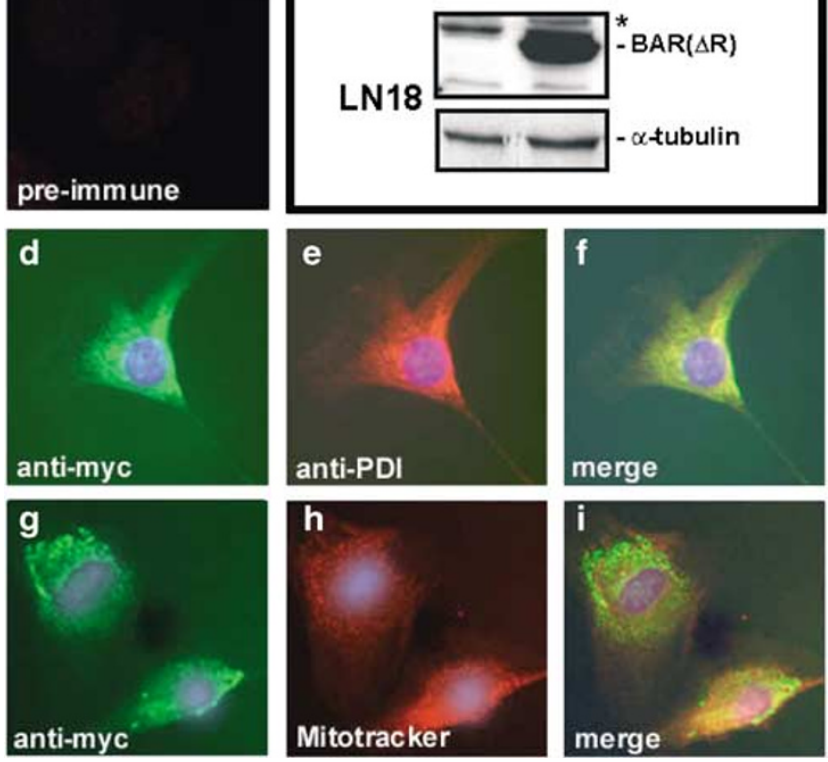

Figure 3 Subcellular localization of BAR. (a,b) Expression pattern of endogenous BAR protein in SK-N-BE(2) human neuroblastoma cells. After fixation and permeabilization, cells were stained with anti-BAR antiserum (1:1000) and Alexa 594 anti-rabbit secondary antibody. As a negative control, cells were incubated with pre-immune serum. (c) Expression control for $\mathrm{BAR}(\Delta \mathrm{R})$ stably transfected cell lines. Whole-cell lysates $(20 \mu \mathrm{g}$ per lane) of control cells and transfectants were subjected to immunoblot analysis $\left({ }^{*}=\right.$ unspecific band). Reprobing the blot with anti- $\alpha$-tubulin antibody served as a loading control. (d-i) Coimmunostaining of BAR and ER or mitochondria. CSM cells were differentiated to neurons, fixed, and permeabilized as outlined in the Methods section. Ectopic BAR expression was detected by staining with anti-myc antibody (d,g). Anti-PDI was used as an ER marker (e), and Mitotracker was used as a mitochondrial marker (h). Merged pictures are shown in (f) and (i)

treated neuronal and astrocytoma cells with staurosporine (STS), an inducer of the intrinsic apoptotic pathway. While BAR overexpressing CSM cells were substantially protected from STS-induced cell death, LN18 cells were not (Figure $4 a, b)$. To evaluate the extent of BAR-mediated protection in CSM cells compared to other antiapoptotic proteins, we performed identical experiments with CSM cells stably transfected with $\mathrm{Bcl}-2 .{ }^{13}$ BAR conferred significant resistance to STS, but the extent of protection was not as large as that afforded by Bcl-2.

These cells were also subjected to serum deprivation as another type of stimulus thought to operate primarily through the mitochondrial cell death pathway. BAR-expressing CSM cells clearly resisted serum withdrawal, whereas the differences in cell survival of LN18 astrocytoma cells did not achieve statistical significance (Figure 4c,d). Again, protection afforded by $\mathrm{Bcl}-2$ was more potent than BAR in CSM cells subjected to serum deprivation.
Next, we tested the extrinsic signalling pathways that are normally induced by activation of death receptors. CSM cells were treated with $\mathrm{TNF} \alpha$, whereas $\mathrm{TNF} \alpha$-resistant $\mathrm{LN} 18$ cells were treated with anti-Fas antibody $\mathrm{CH} 11$. In this cell death model, BAR expression resulted in substantial protection in both CSM and LN18 cells (Figure 4e,f). Interestingly, protection mediated by $\mathrm{BAR}$ and $\mathrm{Bcl}-2$ was of similar potency in CSM cells.

Since BAR predominantly localizes to the ER, and because ER stress is an important cell death stimulus in normal and diseased CNS cells, ${ }^{14}$ we treated the cell lines with thapsigargin, a specific antagonist of the ER $\mathrm{Ca}^{2+}$-ATPase. CSM cells, but not LN18 cells, were significantly protected from thapsigargin-induced cell death by overexpression of BAR (Figure 4g,h). The protection conferred against ER stress by BAR was somewhat weaker than by Bcl-2.

We have previously shown that the wild-type BAR protein and the BAR $\triangle \mathrm{R}$ mutant have similar antiapoptotic activity in 293 T cells. ${ }^{11}$ To confirm that protection of neuronal cells from diverse cell death stimuli can be achieved by full-length BAR as well as $B A R \Delta R$, we transiently transfected CSM cells with a plasmid encoding wildtype BAR or the empty expression vector together with a GFP-encoding plasmid. After performing similar treatments as shown in Figure 4, apoptosis was assessed by counting GFP-positive cells exhibiting apoptotic morphology of DAPI-stained nuclei. The percentages ( \pm S.D.) of apoptosis of CSM cells transfected with full-length BAR or control vector were $24( \pm 4)$ versus $37( \pm 6) \%$ after treatment with staurosporine $(0.01 \mu \mathrm{M}, 12 \mathrm{~h}) ; 19( \pm 2)$ versus $42( \pm 6) \%$ after serum deprivation (16 h); $7( \pm 3)$ versus $20( \pm 4) \%$ after incubation with $\mathrm{TNF} \alpha(50 \mathrm{ng} / \mathrm{ml} ; 12 \mathrm{~h})$; and $16( \pm 5)$ versus $32( \pm 3) \%$ after treatment with thapsigargin $(0.3 \mu \mathrm{M} ; 12 \mathrm{~h})$. These experiments confirmed that overexpressed wild-type $B A R$ and $B A R \triangle R$ have similar antiapoptotic effects in neuronal cells.

In addition to cell death, we examined the effects of BAR expression on caspase activation. After treatment of BARtransfected or control LN18 cells with anti-Fas antibody $\mathrm{CH} 11$, Caspase-3-like protease activity was measured by incubation of lysates with Caspase-3 substrate DEVD-AFC. While control cells showed a prominent increase in DEVD-AFC cleavage, Caspase activity in BAR transfectants remained low (Table 1). In parallel experiments, pro-Caspase-8 cleavage was examined by detection of the active $p 18$ subunit by immunoblot analysis. Cleavage of pro-Caspase- 8 into active subunits was readily detected in control cells even at low concentrations of $\mathrm{CH} 11$ antibody $(0.08 \mu \mathrm{g} / \mathrm{ml})$, while BARoverexpressing cells showed cleavage of pro-Caspase- 8 only at 25-fold higher concentrations of anti-Fas antibody (Figure 5).

Next, we tested if neuronal-lineage cells could be sensitized to apoptosis by downregulating endogenous BAR expression using antisense oligonucleotides. Lipid-mediated transfection of two different antisense oligonucleotides specific for human BAR into SK-N-BE(2) neuroblastoma cells resulted in almost complete loss of BAR mRNA, as assessed by quantitative RTPCR methods after $24 \mathrm{~h}$ (Figure $6 \mathrm{a}$ and data not shown). Since the adapter protein FADD and the cell death protease Caspase-8 participates in the death receptor-induced apoptosis pathways, we tested the effects of downregulating 
Table 1 Caspase-3-like activity in BAR-overexpressing versus control-trans fected cells

\begin{tabular}{lrrrr}
\hline & \multicolumn{2}{c}{ Control } & \multicolumn{2}{c}{ BAR } \\
\cline { 2 - 5 } Min & \multicolumn{1}{c}{ Co. } & \multicolumn{1}{c}{+ CH11 } & \multicolumn{1}{c}{ Co. } & +CH11 \\
\hline 1 & $51 \pm 8$ & $108 \pm 2$ & $73 \pm 1$ & $74 \pm 2$ \\
5 & $38 \pm 5$ & $358 \pm 3$ & $64 \pm 4$ & $79 \pm 7$ \\
10 & $28 \pm 2$ & $586 \pm 11$ & $50 \pm 5$ & $108 \pm 9$ \\
15 & $17 \pm 1$ & $753 \pm 10$ & $46 \pm 7$ & $116 \pm 6$ \\
\hline
\end{tabular}

LN18 cells were incubated without ('Co.') or with anti-Fas antibody $\mathrm{CH} 11$ $(0.5 \mu \mathrm{g} / \mathrm{ml})$ for $6 \mathrm{~h}$. Cell lysates were generated from equivalent numbers of cells and incubated with DEVD-AFC. Cleavage of DEVD-AFC substrate was monitored by measuring fluorescence intensity. Data are presented as mean \pm standard deviation of four consecutive measurements at the indicated times

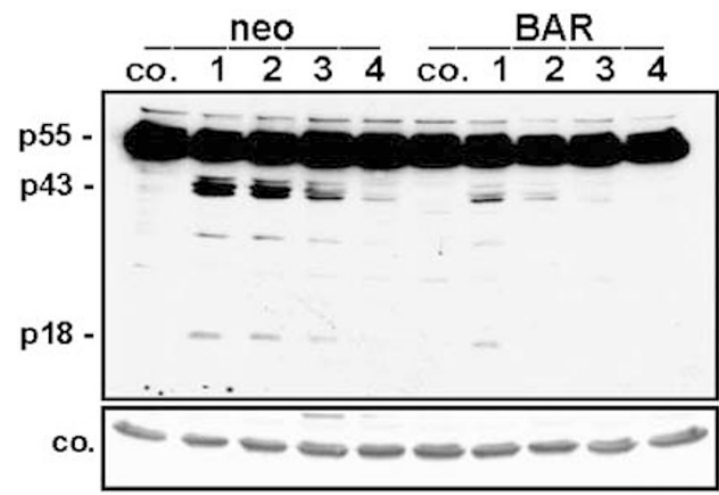

Figure 5 Role of BAR in extrinsic apoptosis. Caspase-8 cleavage after treatment with anti-Fas antibody. LN18 cells were treated with anti-Fas antibody CH11 for $16 \mathrm{~h}$ using (1) $2 \mu \mathrm{g} / \mathrm{ml}$; (2) $0.4 \mu \mathrm{g} / \mathrm{ml}$; (3) $0.08 \mu \mathrm{g} / \mathrm{m}$; or (4) $0.016 \mu \mathrm{g} / \mathrm{ml}$. Cell lysates were subjected to immunoblot analysis with mouse monoclonal antiCaspase-8 antibody (Upstate Biotechnology)

oligonucleotides that are specific for the human BAR sequence. However, antisense-mediated downregulation of the ectopically expressed human BAR protein resulted in resensitization of CSM cells to apoptosis, thereby confirming that the resistant phenotype of BAR-transfected CSM cells was specifically caused by BAR (Figure 6b).

\section{Novel candidate interaction partners of BAR}

BAR protein contains a pseudo-DED, similar to Huntingtininteracting protein (HIP1), HIP1 protein interactor (Hippi), and B-lymphocyte receptor-associated protein 31 (Bap31). We therefore tested BAR for interactions with HIP1, Hippi, and Bap31 by coimmunoprecipitation assays. For these experiments, candidate interacting proteins were transiently coexpressed with BAR in 293 T cells and subsequently coimmunoprecipitated. No interaction was detectable for the DED-containing proteins FADD and PEA15, or for non-DED proteins such as XIAP and Bid (Figure 7a and data not shown). In contrast, HIP1, Hippi, and Bap31 were readily detected in BAR-containing immune complexes (Figure 7a).

Since physiological protein interactions require that the binding partners share the same intracellular compartments, we tested whether the interacting proteins colocalize in immunofluorescence experiments. COS-7 cells were transiently cotransfected with plasmids encoding a GFP-BAR 
a
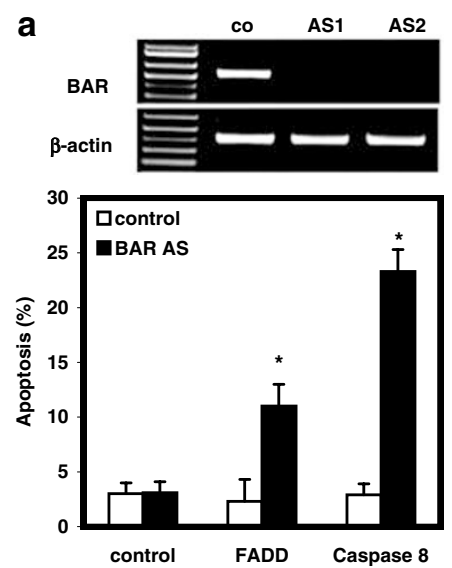

b
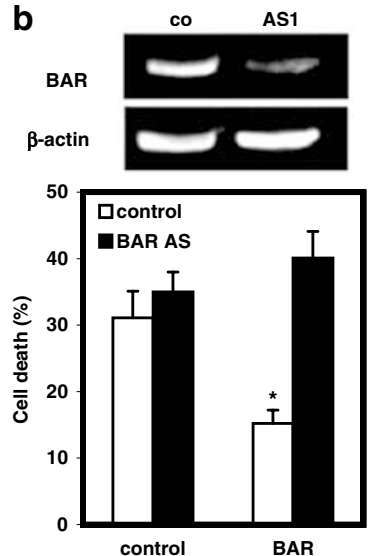

Figure 6 Antisense-mediated reductions in BAR sensitize cells to apoptosis. (a) SK-N-BE(2) human neuroblastoma cells were transfected with two different BAR-specific antisense oligonucleotides (AS1, AS2; $150 \mathrm{nM}$ ). After $24 \mathrm{~h}$, the cells were transiently transfected with FADD or Caspase-8 $(1 \mu \mathrm{g} / \mathrm{ml})$, and apoptotic cell death was assessed after $16 \mathrm{~h}$ by DAPI staining. The data shown are derived from samples treated with antisense oligonucleotide AS1 $(n=4, P=0.05)$, and represent mean \pm S.D. Transfecting cells with AS2 produced similar results (not shown). (b) Downregulation of BAR reverses the resistant phenotype in BARoverexpressing cells. BAR-overexpressing CSM cells were transfected with antisense oligonucleotide AS1 (150 nM) or control oligonucleotide ('co'). After $24 \mathrm{~h}$, cells were cultured with TNF $\alpha(50 \mathrm{ng} / \mathrm{ml})$ for $16 \mathrm{~h}$. Cell death was assessed by trypan blue exclusion assay $(n=4$, mean \pm S.D.; $P=0.05)$

fusion protein (pEGFP-BAR full-length) and FLAG-tagged HIP1, Hippi, Bap31, or FADD. After staining the cells with antiFLAG antibody, the intracellular localization of the proteins was examined by confocal laser-scanning microscopy (Figure 7b). The fluorescence signal of GFP-BAR coincided well with the staining patterns for HIP1, Hippi, and Bap31. In contrast, only a partial overlap between GFP-BAR and FADD (bottom panel) or FLIP (not shown) was observed.

\section{Discussion}

The preservation of neurons is crucial for the functional integrity of the central nervous system. Since cells of neuronal origin are in general irreplaceable, loss of substantial amounts of brain tissue often results in permanent neurological deficits. On a cellular basis, the conservation of neurons for the entire lifespan of the organism is a considerable task, given the many diverse damaging stimuli that can impact neurons throughout life. Since apoptosis is believed to play an important role in the pathological loss of neurons, the expression of proteins that block apoptotic signalling may be decisive for the prevention of neuronal death. Dysregulated apoptotic cell death is held accountable for several diseases of the nervous system, and particularly within the group of neurodegenerative diseases, apoptosis has been implicated as a potentially crucial pathogenetic mechanism. ${ }^{1,15-18}$ Despite the considerable progress in the knowledge about pathological features of neurological diseases, the therapeutic options are currently limited. Therefore, a need exists for additional insight into the mechanisms of how life and death decisions are regulated in adult neurons.

Two major routes of induction of apoptosis are the intrinsic and extrinsic pathways. The intrinsic pathway depends mainly on mitochondrial events, such as release of cytochrome $c$, leading to oligomerization of the CARD-containing protein Apaf- 1 and activation of the CARD-carrying protease Caspase 9 , and then subsequent activation of downstream caspases. ${ }^{19}$ In contrast, the extrinsic cell death pathway is induced by TNF-family death receptors and involves the formation of membrane-bound signalling complexes consisting of the cytosolic death domains (DDs) of death receptors, adaptor proteins, and DED-containing caspases. ${ }^{20}$

BAR was described as a protein at the crossroads of intrinsic and extrinsic apoptosis pathways. ${ }^{11}$ Evidence has been presented that complexes of $\mathrm{Bcl}-2 / \mathrm{Bcl}-\mathrm{X}_{\mathrm{L}}$ and BAR can sequester active Caspase- 8 subunits on intracellular membranes, thereby neutralizing the active Caspase- 8 fragments and preventing cleavage of substrates. ${ }^{12}$ Despite these insights into the possible molecular mechanism of BAR, little has been known regarding the physiological functions of this protein in vivo. Therefore, we sought to define the anatomic location of the BAR protein and to interrogate its function as a suppressor of variant apoptotic stimuli. Interestingly, tissue expression of BAR is highly restricted, with brain tissue representing the predominant site of expression, followed by intestine, epidermis, testis, and little or no expression in other organs. Furthermore, immunohistochemical analysis revealed that BAR is expressed almost exclusively by neurons, but not by astrocytes or other cells in the brain, such as microglia. Interestingly, peripheral neuronal cells such as sympathetic ganglion cells also expressed high levels of BAR, suggesting that BAR expression is a general feature of neuronal cells and independent of their anatomical location. The staining of BAR in autonomic ganglion cells within the bowel wall and the absence of BAR in intestinal epithelial or stromal cells provides an explanation for the moderate BAR expression observed in small intestine by immunoblotting.

Immunofluorescence studies indicated that BAR predominantly localizes to the ER. Interestingly, only a minor fraction of the total BAR colocalized with mitochondria in neural cell lines, although it has been reported that the BAR-mediated sequestration of active Caspase- 8 takes place at mitochondrial membranes in MCF-7 cells. ${ }^{12}$ Thus, BAR distribution between ER and mitochondria could differ depending on the cell type, particularly because the ER and the mitochondrial network can coalesce into a continuous membrane structure. ${ }^{21}$ An ER-localization for BAR is also consistent with its ability to coimmunoprecipitate with Bap31, which is an ERresident protein. ${ }^{22}$ It is conceivable that BAR could have ERspecific cytoprotective functions, given its ability to suppress cell death induced by ER stress.

For our studies of BAR in neuronal cells, we took advantage of the cell line CSM14.1. These cells originate from rat nigral neurons immortalized by expression of temperature-sensitive SV40 large T antigen. CSM cells show a high proliferative rate at permissive temperature $\left(32^{\circ} \mathrm{C}\right)$, while inactivation of large $\mathrm{T}$ antigen at nonpermissive temperature $\left(39^{\circ} \mathrm{C}\right)$ induces neuronal differentiation. ${ }^{13,23}$ Overexpression of $\mathrm{Bcl}-2$ in these cells resulted in protection from a variety of death stimuli. ${ }^{13}$ Similarly, overexpression of wild-type BAR as well as BAR $\Delta R$ rendered CSM cells more resistant to a broad range of 
a
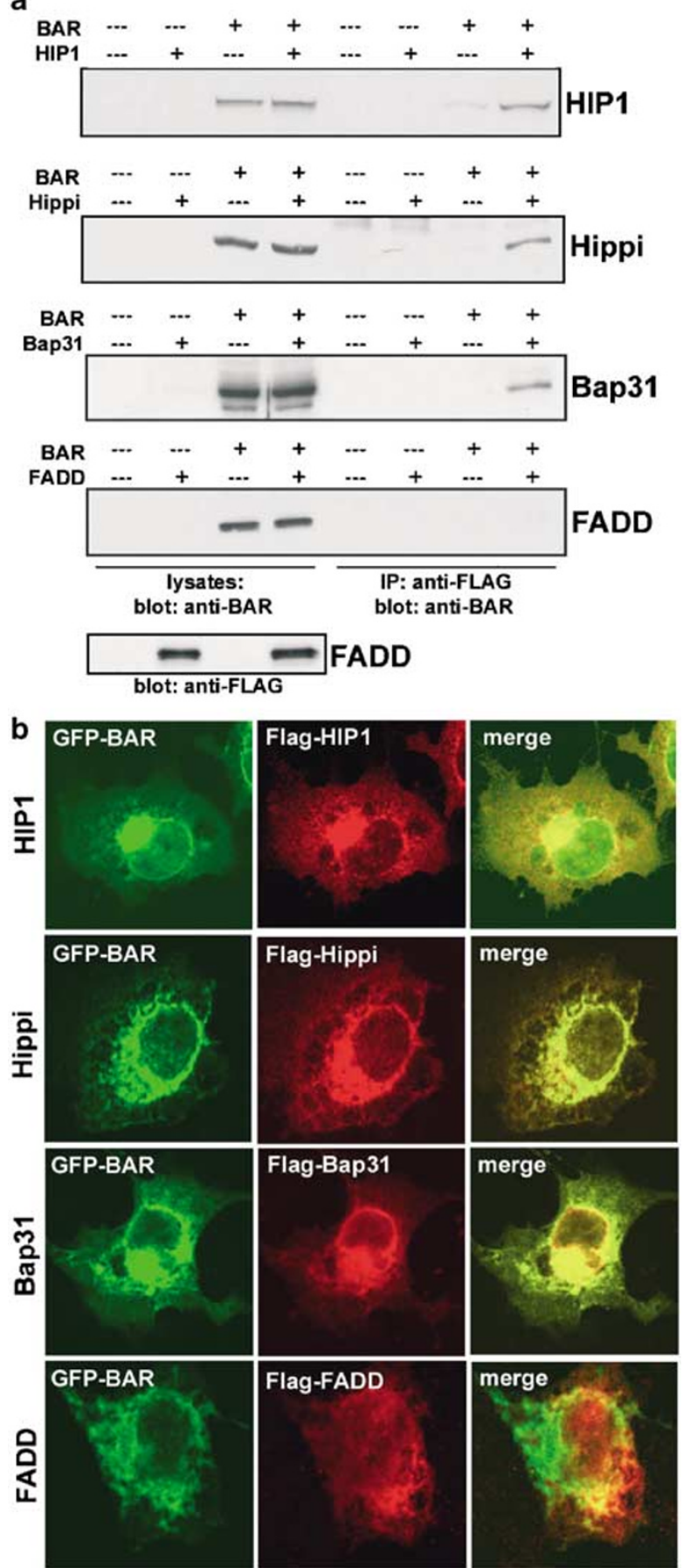

Figure 7 Interaction of BAR with HIP1, Hippi, and Bap31. (a) Myc-BAR and FLAG-tagged HIP1, Hippi, Bap31, and FADD were expressed in $293 \mathrm{~T}$ cells by transient transfection. After immunoprecipitation of FLAG-tagged proteins, protein complexes were resolved by SDS-PAGE and transferred to nitrocellulose. The membrane was incubated with BAR-specific antiserum $(1: 3000)$. Shown are the expression controls for BAR in cell lysates (left four lanes) and immunoprecipitates (right four lanes). Membranes were reprobed with antiFLAG antibody to demonstrate sufficient and equal expression of FLAG-tagged proteins as demonstrated for FADD (bottom panel and not shown). (b) Colocalization of BAR with HIP1, Hippi, and Bap31. COS-7 cells were transfected with plasmids encoding GFP-BAR, FLAG-HIP1, FLAG-Hippi, FLAG-Bap31, and FLAG-FADD. At $20 \mathrm{~h}$ post-transfection, cells were fixed in $2 \%$ formaldehyde, stained with a monoclonal antibody recognizing the FLAG epitope followed by an Alexa 594-labelled secondary antibody, and visualized by laser confocal microscopy. The overlay of the images is shown in the right column cytotoxic stimuli. We chose several cell death assays to model various types of stress and injury known to affect neurons in vivo. Staurosporine and $\operatorname{TNF} \alpha$ were used to trigger the intrinsic and extrinsic pathways for apoptosis, respectively. For example, intrinsic, mitochondria-dependent apoptosis could play an important role in the pathogenesis of Parkinson's disease, since Bax gene ablation inhibited neuronal apoptosis in an MPTP mouse model of Parkinson's disease. ${ }^{24}$ Similarly, extrinsic apoptosis pathways triggered by TNF $\alpha$ could play a role during the progression phase of stroke, where cytokine elaboration occurs in the peri-infarct zone after a focal ischemia. ${ }^{25}$ Moreover, ER stress leading to induction of cell death in the brain has gained more attention in recent years. In the case of Alzheimer's disease, increasing evidence suggests that mutations in the presenilin-1 gene and perturbed ER calcium homeostasis render neurons more susceptible to damaging insults. ${ }^{26}$ In this regard, BAR expression in CSM cells conferred resistance to thapsigargin, which is an irreversible inhibitor of $\mathrm{Ca}^{2+}$-ATPase of the ER. ${ }^{27}$ Finally, serum deprivation is a well-described and common experimental in vitro model for a nonsupportive microenvironment. Pathological conditions such as stroke and cerebral edema might result in an undersupply of essential nutrients.

In contrast to neuronal cells, BAR-overexpressing LN18 astrocytoma cells were only protected from death receptormediated cell death but not from the other cytotoxic stimuli. Similar effects were seen with a second astrocytoma cell line, LN229 (not shown). Differences in the expression of interaction partners of BAR or variations in the BAR-relevant pathways may account for the varying effects of BAR overexpression in these cell lines. Future investigations are needed to pinpoint the responsible proteins and to test a wider panel of cell lines for responsiveness to BAR-mediated protection.

Interestingly, BAR expression resulted not only in an inhibition of Caspase-3 activation but also in a decrease of the Caspase-8 active subunits (Table 1; Figure 5). BAR has been shown to sequester active Caspase- 8 subunits, ${ }^{12}$ but this does not inhibit the generation of the p43 and p18 cleavage products as seen in our experiments (Figure 5). Therefore, our findings suggest that BAR additionally affects the further processing of Caspase-8. It is known that active Caspase-8 can induce the mitochondrial apoptosis pathway via cleavage of Bid, resulting in cytochrome $c$ release and Apaf1-mediated oligomerization of Caspase-9. These mitochondrial events, however, can in turn lead to cleavage and activation of Caspase-8, which establishes a positive amplification loop. ${ }^{28}$ Thus, BAR-mediated sequestration of Caspase- 8 active subunits could prevent the mitochondrialdependent amplification of Caspase-8 activation.

In addition to overexpression experiments, we interrogated the function of BAR using antisense oligonucleotides to reduce endogenous BAR expression. Since the available antisense oligonucleotides were specific for human BAR, they could not be used to sensitize rat CSM cells by downregulating endogenous BAR, and thus we chose a human neuroblastoma cell line that contains high levels of BAR protein. Transfection of neuroblastoma cells with antisense oligonucleotides downregulated BAR mRNA almost completely. By monitoring the BAR protein levels after antisense 


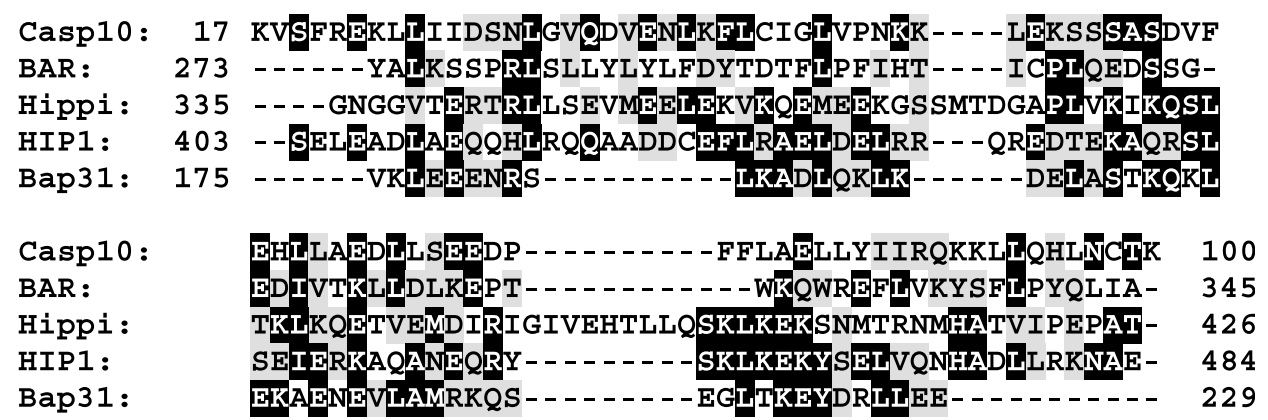

Figure 8 Comparison of pseudo-DEDs. An alignment is presented of the classical DED(1) of Caspase-10 and the pseudo-DEDs of BAR, Hippi, HIP1, and Bap31. At the beginning and the end of each sequence, the relative position of the domain in the molecule is shown. The amino-acid sequences were obtained from GenBank (NCBI) and aligned using the ClustalX program. Identical and similar residues are indicated in black and gray, respectively

oligonucleotide treatment, the half-life of the BAR protein was $\sim 6 \mathrm{~h}$ (not shown). Antisense-mediated BAR suppression sensitized neuroblastoma cells to proapoptotic stimuli, including transfection with FADD or Caspase-8. These experiments thus confirm the antiapoptotic properties of BAR by an independent method.

Given the broad range of antiapoptotic activity of BAR, it is entirely possible that interactions with proteins other than $\mathrm{Bcl}-$ 2 and Caspase- 8 are important for its cytoprotective functions. Since BAR contains a variant DED domain similar to those found in HIP1, Hippi, and Bap31 (Figure 8), we tested BAR for interactions with these and selected other apoptosis-related proteins by coimmunoprecipitation assays. Interestingly, the three proteins containing pseudo-DEDs exhibit binding to BAR: HIP1, Hippi, and Bap31. In contrast, no interaction was detected with the classical DED-containing proteins, FADD and PEA15. Moreover, BAR showed extensive colocalization with HIP1, Hippi, and Bap31 in confocal microscopy studies, whereas FADD or FLIP did not clearly overlap with BAR. These findings raise the intriguing possibility that BAR interacts with other pseudo-DED-containing proteins, some of which are reported to be involved in regulating neuronal cell death. The $110 \mathrm{kDa}$ protein HIP1, for example, binds to Huntingtin, which suffers poly-glutamine expansions in Huntington's disease. ${ }^{29,30}$ Recently, it has been suggested that mutated Huntingtin releases the HIP1 protein, which then is free to interact with another novel protein, Hippi. HIP1 and Hippi reportedly form a complex together with pro-Caspase-8, thereby triggering caspase activation and cell death. ${ }^{10}$ In addition, Bap31 is a transmembrane protein that resides mainly in the ER and which also contains a pseudo-DED. ${ }^{22}$ Similar to BAR, Bap31 reportedly binds Bcl-2 as well as proCaspase-8. ${ }^{22}$ Interestingly, Bap31 expression is highest in neurons, lymphocytes, and endocrine cells. ${ }^{31}$ Future investigations will reveal to what extent the interactions of these variant DED-containing proteins are relevant for BARmediated neuroprotection.

\section{Materials and Methods}

\section{Transfections}

The rat nigrostriatal cell line CSM 14. 1, immortalized by introduction of the temperature-sensitive SV40 large $\mathrm{T}$ antigen, and a stably $\mathrm{Bcl}-2$ transfected subline of these cells were kindly provided by Dale
Bredesen. ${ }^{13}$ Cells were maintained in Dulbecco's modified Eagle's medium (DMEM) supplemented with 10\% fetal bovine serum (FBS), $1 \mathrm{mM}$ L-glutamine, $100 \mathrm{U} / \mathrm{ml}$ penicillin, and $100 \mu \mathrm{g} / \mathrm{ml}$ streptomycin sulfate either at a permissive temperature of $32^{\circ} \mathrm{C}$ or nonpermissive temperature of $39^{\circ} \mathrm{C}^{13}$ For stable transfection, $50-70 \%$ confluent CSM cells in six-well plates were transfected with pcDNA3-myc-BAR $\Delta R$ and pBabe puro using Gene Porter II (Gene Therapy Systems). LN-18 human glioblastoma cells were transfected with pcDNA3 neo (control), or pcDNA3-myc-BAR $\Delta \mathrm{R}^{11}$ plasmids using Lipofectamine PLUS (Life Technologies). After 2 days, complete medium containing puromycin $(4 \mu \mathrm{g} / \mathrm{ml})$ (Sigma) or $\mathrm{G} 418$ $(700 \mu \mathrm{g} / \mathrm{ml})$ (Omega Scientific), respectively, was used to select stably transfected cells. Single clones were obtained 4-8 weeks after transfection by limiting-dilution cloning. For transient transfections, $293 \mathrm{~T}$, SK-N-BE(2), COS-7, and CSM 14.1 cells were transfected using Lipofectamine PLUS (Life Technologies) with plasmid DNA encoding mycBAR (full-length), myc-BAR $\triangle \mathrm{R}, \mathrm{FADD}$, or pro-Caspase-8, and, in some experiments, green fluorescent protein (GFP) marker plasmid pEGFP (Clontech). In each experiment, the total amount of DNA was normalized using pcDNA3.

\section{Immunohistochemistry}

The distribution of BAR protein expression in human organ tissues was assessed in single paraffin sections or tissue microarray slides derived from human biopsy and autopsy material. Dewaxed tissue sections were exposed to a $1: 3000(\mathrm{v} / \mathrm{v})$ dilution of polyclonal antiserum raised against an immunogen consisting of a recombinant $\mathrm{N}$-terminal portion (1-139 aa) of the human BAR protein (AR-48). In addition, a rabbit polyclonal antibody was raised against an N-terminal peptide (3-21 aa: CEPQKSYVNTMDLERDEPLK) conjugated with carrier proteins (AR-47). Specificity was confirmed by using preimmune serum or anti-BAR antiserum preadsorbed with recombinant BAR immunogen $(10 \mu \mathrm{g} / \mathrm{ml})$ or the $\mathrm{N}$ terminal peptide, respectively. The sections were immunostained using a diaminobenzidine (DAB)-based detection method, employing either an avidin-biotin complex reagent (Vector Laboratories) or the Envision-PlusHorse Radish Peroxidase (HRP) system (DAKO) using an automated immunostainer (Dako Universal Staining System).

\section{Immunofluorescence and confocal microscopy}

For immunofluorescence, control and stably transfected CSM cells expressing myc-BAR $\Delta R$ were cultured in eight-well, covered chamber slides (Nalge Nunc) at 32 or $39^{\circ} \mathrm{C}$. After incubation with $25 \mathrm{nM}$ Mitotracker Red (Molecular Probes), cells were washed and fixed in PBS containing 
$4 \%$ paraformaldehyde for $15 \mathrm{~min}$ at room temperature, followed by several washing steps in PBS. Permeabilization was performed in $0.3 \%$ Triton X$100 /$ PBS for 5 min with subsequent preblocking in PBS containing $2 \%$ normal goat serum. Cells were incubated for $1 \mathrm{~h}$ at $4^{\circ} \mathrm{C}$ in blocking solution containing the following primary antibodies: anti-BAR (1:1000; AR-48), anti-myc (1:100; Santa Cruz), or anti-protein disulfide isomerase (PDI, $1: 250$; Stressgene). After washing three times in PBS and incubation with FITC-conjugated secondary anti-mouse or anti-rabbit antibody $(1: 50$; Dako) or Alexa 594 goat anti-rabbit $\operatorname{lgG} \mathrm{F}\left(\mathrm{ab}^{\prime}\right)_{2}$ secondary antibody (1:200; Molecular Probes) for $1 \mathrm{~h}$ at room temperature, slides were covered with Vectashield mounting medium with or without $1.5 \mu \mathrm{g} / \mathrm{ml} 4,6$ diamidino-2-phenylindole (DAPI) (Vector Laboratories) and sealed with Cytoseal 60 mounting medium (Stephens Scientific). For confocal microscopy, COS-7 cells were cultured in eight-well, covered chamber slides (Nalge Nunc) $\left(10^{4}\right.$ cells per chamber), transfected with various plasmids (pEGFP-BAR, pEGFP, pcDNA3-FLAG-HIP1, pcDNA3-FLAGHippi, pcDNA3-FLAG-Bap31, and pcDNA3-FLAG-FADD) using Lipofectamine PLUS reagent, and incubated at $37^{\circ} \mathrm{C}$ for $20 \mathrm{~h}$. After staining with a monoclonal mouse anti-FLAG M2 antibody (Sigma) and Alexa 594 goat anti-mouse secondary antibody, the cells were imaged by confocal microscopy using a Bio-Rad MRC 1024 instrument.

\section{Immunoblot analysis}

Lysates from adult human tissues were purchased from Imgenex (San Diego, CA, USA). Lysates from LN18 cells and CSM cells were generated by resuspending cell pellets in lysis buffer $(150 \mathrm{mM} \mathrm{NaCl}, 20 \mathrm{mM}$ Tris (pH 7.4), $0.2 \% \mathrm{NP}-40$ ) containing $1 \mathrm{mM}$ phenylmethylsulfonyl fluoride, and a protease inhibitor mixture (Roche). Proteins were resolved by SDS-PAGE and transferred onto nitrocellulose membranes. After blocking with $5 \%$ skim milk in PBS at room temperature for $1 \mathrm{~h}$, blots were incubated with various primary antibodies including polyclonal antisera against BAR (AR48; 1:3000), Bap31 (1:3000; kindly provided by Gordon Shore), and monoclonal antibodies against Caspase-8 (1:1000; clone 5F7, Upstate Biotechnology), myc (1: 100; Santa Cruz), and FLAG (1:1000; Sigma), followed by HRP-conjugated anti-mouse or anti-rabbit IgG (Biorad) secondary antibodies. Bound antibodies were visualized using an enhanced chemiluminescence (ECL) detection system (Amersham).

\section{Cell death assays}

For cytotoxicity assays, cells were plated in 96-well plates, cultured for $24 \mathrm{~h}$, and then incubated for $16 \mathrm{~h}$ with staurosporine $(0.01 \mu \mathrm{M}), \mathrm{TNF} \alpha$ $(50 \mathrm{ng} / \mathrm{ml})$, anti-Fas antibody $\mathrm{CH} 11(0.2 \mu \mathrm{g} / \mathrm{ml})(\mathrm{MBL})$, or thapsigargin $(0.3 \mu \mathrm{M})$. Cell death was assessed by Trypan blue exclusion assay. Additionally, the percentage of surviving cells was assessed by staining with crystal violet, as previously described in detail. ${ }^{32}$ Cell death by serum deprivation was induced as published previously. ${ }^{13}$ Cell survival was assessed by crystal violet staining after 2 days of serum starvation. Experiments were repeated four times.

\section{Synthesis and transfection of antisense oligonucleotides}

Second-generation 2'-O-methoxyethyl/DNA chimeric phosphorothioate oligonucleotides that inhibit BAR expression were identified following evaluation of 78 different oligonucleotides designed to bind to human BAR mRNA. Oligonucleotides were synthesized and purified as previously described. ${ }^{33}$ A549 cells were plated in 96-well plates and treated with
$150 \mathrm{nM}$ oligonucleotide in the presence of $3.75 \mu \mathrm{g} / \mathrm{ml}$ Lipofectin reagent (Life Technologies) for $4 \mathrm{~h}$ at $37^{\circ} \mathrm{C}$. Cells were incubated overnight in the absence of oligonucleotide. Total RNA was extracted using an RNeasy Mini prep kit (Qiagen) according to the manufacturer's protocol. Gene expression was analyzed using quantitative RT-PCR. The following primer/probe sets were used. Forward primer: 5'-GTGGAGAGAGTTCCTGGTCAAATAC-3', reverse primer: 5'-CCAACCAGTCCCAAGCAAA-3'. Several oligonucleotides were identified that inhibited BAR expression by greater than $70 \%$. Isis 143770 (AS1; 5'TCCAGCCGCCGGAGTTCCTT-3') and Isis 143780 (AS2; 5'TCTCTATGCCTATGACAGAA-3' $)$ were selected for additional studies. (Underlined text represents nucleosides containing the 2' -O-methoxyethyl modifications.) Isis 129689 (5'-GAGGTCTCGACTTACCCGCT-3') was used as a control. SK-N-BE(2) or CSM cells were transfected with antisense oligonucleotides prior to inducing cell death as above. Antisense oligonucleotides or the control oligonucleotide were transfected at a concentration of $150 \mathrm{nM}$ according to the Lipofectin method (Life Technologies). After $24 \mathrm{~h}, \mathrm{CSM}$ cells were treated with TNF $\alpha(50 \mathrm{ng} / \mathrm{ml})$ for $16 \mathrm{~h}$ and cell death was measured by trypan blue exclusion assay. SK$\mathrm{N}-\mathrm{BE}(2)$ cells were transiently transfected with FADD or Caspase-8 $(1 \mu \mathrm{g} /$ $\mathrm{ml}$ ), and $0.2 \mu \mathrm{g} / \mathrm{ml}$ of GFP marker plasmid pEGFP (Clontech). At $16 \mathrm{~h}$ post-transfection, both adherent and floating cells were collected, fixed, and stained with $0.1 \mu \mathrm{g} / \mathrm{ml}$ DAPI. The percentage of apoptotic cells was determined by counting the GFP-positive cells exhibiting nuclear condensation and/or fragmentation.

\section{Caspase activity assay}

Caspase-3-like activity was measured by DEVD-AFC cleavage. Briefly, $10^{4}$ cells were plated in 96-well plates, adhered for $24 \mathrm{~h}$, and incubated for $6 \mathrm{~h}$ with anti-Fas antibody $\mathrm{CH} 11(0.5 \mu \mathrm{g} / \mathrm{ml} ; \mathrm{MBL})$. The cells were suspended in lysis buffer $(60 \mathrm{mM} \mathrm{NaCl}, 5 \mathrm{mM}$ Tris/ $\mathrm{HCl}, 2.5 \mathrm{mM}$ EDTA, $0.25 \% \mathrm{NP} 40$ ) for $10 \mathrm{~min}$. Subsequently, the fluorogenic Caspase-3 substrate acetyl-aspartyl-glutamyl-valinyl-aspartyl-7-amino-4-trifluoromethyl-coumarin (Ac-DEVD-AFC; $100 \mu \mathrm{M}$ ) was added to the lysates. Caspase activity was assayed by using a fluorometer plate reader, measuring release of fluorescent AFC.

\section{Co-immunoprecipitation assays}

Cell lysates were precleared and incubated with $20 \mu$ anti-FLAG antibody M2-conjugated agarose (Sigma) at $4{ }^{\circ} \mathrm{C}$ for $8 \mathrm{~h}^{32}$ Controls included immunoprecipitations (IPS) performed using an equivalent amount of normal mouse IgG in combination with Protein G-Sepharose 4B. After extensive washing with lysis buffer, immune complexes were fractionated by SDS-PAGE and transferred to nitrocellulose for immunoblotting using polyclonal antisera against BAR (1:3000; AR-48) or monoclonal mouse anti-FLAG (1:1000; Sigma) antibody, followed by HRPase-conjugated antibodies.

\section{Acknowledgements}

This work was supported by a fellowship from the Emmy-NoetherProgramm of the Deutsche Forschungsgemeinschaft to W. Roth (Ro 2270/ $1-1)$ and by grants from the NIH (AG15393 and NS 36821). We thank D. Bredesen for providing CSM 14.1 and CSM 14.1-Bcl-2 cells, M. Weller for glioma cell lines LN18 and LN229, G. Shore for anti-Bap31 antibody, M. Hayden for the HIP1 plasmid, and F. Bennett for antisense oligonucleotides. 


\section{References}

1. Mattson MP (2000) Apoptosis in neurodegenerative disorders. Nat. Rev. Mol. Cell. Biol. 1: 120-129

2. Robertson GS, Crocker SJ, Nicholson DW and Schulz JB (2000) Neuroprotection by the inhibition of apoptosis. Brain Pathol. 10: 283-292

3. Dirnagl U, ladecola C and Moskowitz MA (1999) Pathobiology of ischaemic stroke: an integrated view. Trends Neurosci. 22: 391-397

4. Yuan J and Yankner BA (2000) Apoptosis in the nervous system. Nature 407 802-809

5. Deveraux QL, Roy N, Stennicke HR, Van Arsdale T, Zhou Q, Srinivasula SM, Alnemri ES, Salvesen GS and Reed JC (1998) IAPs block apoptotic events induced by caspase-8 and cytochrome $c$ by direct inhibition of distinct caspases. Embo J. 17: 2215-2223

6. Perrelet D, Ferri A, Listen P, Muzzin P, Korneluk RG and Kato AC (2002) IAPs are essential for GDNF-mediated neuroprotective effects in injured motor neurons in vivo. Nat. Cell. Biol. 4: 175-179

7. Barker V, Middleton G, Davey F and Davies AM (2001) TNFalpha contributes to the death of NGF-dependent neurons during development. Nat. Neurosci. 4: 1194-1198

8. New DR, Maggirwar SB, Epstein LG, Dewhurst S and Gelbard HA (1998) HIV-1 Tat induces neuronal death via tumor necrosis factor-alpha and activation of non- $N$-methyl-D-aspartate receptors by a NFkappaB-independent mechanism. J. Biol. Chem. 273: 17852-17858

9. Kaul M, Garden GA and Lipton SA (2001) Pathways to neuronal injury and apoptosis in HIV-associated dementia. Nature 410: 988-994

10. Gervais FG, Singaraja R, Xanthoudakis S, Gutekunst CA, Leavitt BR, Metzler M, Hackam AS, Tam J, Vaillancourt JP, Houtzager V, Rasper DM, Roy S Hayden MR and Nicholson DW (2002) Recruitment and activation of caspase-8 by the Huntingtin-interacting protein Hip-1 and a novel partner Hippi. Nat. Cell. Biol. 4: 95-105

11. Zhang H, Xu Q, Krajewski S, Krajewska M, Xie Z, Fuess S, Kitada S, Godzik A and Reed JC (2000) BAR: an apoptosis regulator at the intersection of caspases and Bcl-2 family proteins. Proc. Natl. Acad. Sci. USA 97: 2597-2602

12. Stegh AH, Barnhart BC, Volkland J, Algeciras-Schimnich A, Ke N, Reed JC and Peter ME (2002) Inactivation of caspase-8 on mitochondria of $\mathrm{Bcl}-\mathrm{xL}$ expressing MCF7-Fas cells: role for the bifunctional apoptosis regulator protein. J. Biol. Chem. 277: 4351-4360

13. Zhong LT, Sarafian T, Kane DJ, Charles AC, Mah SP, Edwards RH and Bredesen DE (1993) bcl-2 inhibits death of central neural cells induced by multiple agents. Proc. Natl. Acad. Sci. USA 90: 4533-4537

14. LaFerla FM (2002) Calcium dyshomeostasis and intracellular signalling in Alzheimer's disease. Nat. Rev. Neurosci. 3: 862-872

15. Masliah E, Mallory M, Alford M, Tanaka S and Hansen LA (1998) Caspase dependent DNA fragmentation might be associated with excitotoxicity in Alzheimer disease. J. Neuropathol. Exp. Neurol. 57: 1041-1052

16. Sawa A, Wiegand GW, Cooper J, Margolis RL, Sharp AH, Lawler Jr JF, Greenamyre JT, Snyder SH and Ross CA (1999) Increased apoptosis of Huntington disease lymphoblasts associated with repeat length-dependent mitochondrial depolarization. Nat. Med. 5: 1194-1198

17. Martin LJ, Price AC, Kaiser A, Shaikh AY and Liu Z (2000) Mechanisms for neuronal degeneration in amyotrophic lateral sclerosis and in models of motor neuron death (review). Int. J. Mol. Med. 5: 3-13
18. Friedlander RM (2003) Apoptosis and caspases in neurodegenerative diseases (review). N. Engl. J. Med. 348: 1365-1375

19. Green DR and Reed JC (1998) Mitochondria and apoptosis. Science 281: 1309-1312

20. Ashkenazi A and Dixit VM (1998) Death receptors: signaling and modulation. Science 281: 1305-1308

21. Rizzuto R, Pinton P, Carrington W, Fay FS, Fogarty KE, Lifshitz LM, Tuft RA and Pozzan T (1998) Close contacts with the endoplasmic reticulum as determinants of mitochondrial $\mathrm{Ca}^{2+}$ responses. Science 280: 1763-1766

22. Ng FW, Nguyen M, Kwan T, Branton PE, Nicholson DW, Cromlish JA and Shore GC (1997) p28 Bap31, a Bcl-2/Bcl-XL- and procaspase-8-associated protein in the endoplasmic reticulum. J. Cell. Biol. 139: 327-338

23. Kermer P, Krajewska M, Zapata JM, Takayama S, Mai J, Krajewski S and Reed $\mathrm{JC}$ (2002) Bag1 is a regulator and marker of neuronal differentiation. Cell Death Differ. 9: 405-413

24. Vila M, Jackson-Lewis V, Vukosavic S, Djaldetti R, Liberatore G, Offen D, Korsmeyer SJ and Przedborski S (2001) Bax ablation prevents dopaminergic neurodegeneration in the 1-methyl-4-phenyl-1,2,3,6-tetrahydropyridine mouse model of Parkinson's disease. Proc. Natl. Acad. Sci. USA 98: $2837-2842$

25. Hallenbeck JM (2002) The many faces of tumor necrosis factor in stroke. Nat. Med. 8: 1363-1368

26. Vito $P$, Lacana $E$ and D'Adamio $L$ (1996) Interfering with apoptosis: $\mathrm{Ca}(2+)$ binding protein ALG-2 and Alzheimer's disease gene ALG-3. Science 271: 521-525

27. Thastrup O, Cullen PJ, Drobak BK, Hanley MR and Dawson AP (1990) Thapsigargin, a tumor promoter, discharges intracellular $\mathrm{Ca} 2+$ stores by specific inhibition of the endoplasmic reticulum Ca2(+)-ATPase. Proc. Natl. Acad. Sci. USA 87: 2466-2470

28. Slee EA, Harte MT, Kluck RM, Wolf BB, Casiano CA, Newmeyer DD, Wang HG, Reed JC, Nicholson DW, Alnemri ES, Green DR and Seamus JM (1999) Ordering the cytochrome $c$-initiated caspase cascade: hierarchical activation of caspases-2, $-3,-6,-7,-8$, and -10 in a caspase-9-dependent manner. J. Cell. Biol. 144: 281-292

29. Kalchman MA, Koide HB, McCutcheon K, Graham RK, Nichol K, Nishiyama K, Kazemi-Esfarjani P, Lynn FC, Wellington C, Metzler M, Goldberg YP, Kanazawa I, Gietz RD and Hayden MR (1997) HIP1, a human homologue of $S$. cerevisiae Sla2p, interacts with membrane-associated huntingtin in the brain. Nat. Genet. 16: 44-53

30. Wanker EE, Rovira C, Scherzinger E, Hasenbank R, Walter S, Tait D, Colicelli J and Lehrach H (1997) HIP-I: a huntingtin interacting protein isolated by the yeast two-hybrid system. Hum. Mol. Genet. 6: 487-495

31. Manley HA and Lennon VA (2001) Endoplasmic reticulum membrane-sorting protein of lymphocytes (BAP31) is highly expressed in neurons and discrete endocrine cells. J. Histochem. Cytochem. 49: 1235-1243

32. Roth W, Stenner-Liewen F, Pawlowski K, Godzik A and Reed JC (2002) Identification and characterization of DEDD2, a death effector domaincontaining protein. J. Biol. Chem. 277: 7501-7508

33. McKay RA, Miraglia LJ, Cummins LL, Owens SR, Sasmor H and Dean NM (1999) Characterization of a potent and specific class of antisense oligonucleotide inhibitor of human protein kinase $\mathrm{C}$-alpha expression. J. Biol. Chem. 274: 1715-1722 